\title{
Twenty years of active bat rabies surveillance in Germany: a detailed analysis and future perspectives
}

\author{
J. SCHATZ ${ }^{1}$, B. OHLENDORF ${ }^{2}$, P. BUSSE ${ }^{3}$, G. PELZ ${ }^{4}$, D. DOLCH ${ }^{5}$, \\ J. TEUBNER ${ }^{5}$, J. A. ENCARNAÇÃO ${ }^{6}$, R.-U. MÜHLE ${ }^{7}$, M. FISCHER ${ }^{1}$, \\ B. HOFFMANN ${ }^{1}$, L. KWASNITSCHKA ${ }^{1}$, A. BALKEMA-BUSCHMANN ${ }^{1}$, \\ T. C. METTENLEITER ${ }^{1}$, T. MÜLLER ${ }^{1}$ AND C. M. FREULING ${ }^{1 *}$ \\ ${ }^{1}$ Friedrich-Loeffler-Institut, Federal Research Institute for Animal Health, Institute of Molecular Biology, \\ WHO Collaborating Centre for Rabies Surveillance and Research, Greifswald-Insel Riems, Germany \\ ${ }^{2}$ Biosphärenreservat Karstlandschaft Südharz, Landesreferenzstelle für Fledermausschutz Sachsen-Anhalt, Roßla, \\ Germany \\ ${ }^{3}$ Arbeitskreis Fledermäuse Sachsen-Anhalt e.V., Stolberg, Germany \\ ${ }^{4}$ Landesfachausschuss Säugetierkunde Berlin-Brandenburg, Potsdam, Germany \\ ${ }^{5}$ Landesamt für Umwelt, Gesundheit und Verbraucherschutz Land Brandenburg, Naturschutzstation Zippelsförde, \\ Zippelsförde, Germany \\ ${ }^{6}$ Justus-Liebig-University of Giessen, Mammalian Ecology Group, Department of Animal Ecology and \\ Systematics, Giessen, Germany \\ ${ }^{7}$ University of Potsdam, Department of Animal Ecology, Potsdam, Germany
}

Received 27 May 2013; Final revision 31 July 2013; Accepted 14 August 2013; first published online 6 September 2013

\section{SUMMARY}

In Germany, active bat rabies surveillance was conducted between 1993 and 2012. A total of 4546 oropharyngeal swab samples from 18 bat species were screened for the presence of EBLV-1-,

EBLV-2- and BBLV-specific RNA. Overall, $0 \cdot 15 \%$ of oropharyngeal swab samples tested EBLV-1 positive, with the majority originating from Eptesicus serotinus. Interestingly, out of seven

RT-PCR-positive oropharyngeal swabs subjected to virus isolation, viable virus was isolated from a single serotine bat (E. serotinus). Additionally, about 1226 blood samples were tested serologically, and varying virus neutralizing antibody titres were found in at least eight different bat species. The detection of viral RNA and seroconversion in repeatedly sampled serotine bats indicates long-term circulation of the virus in a particular bat colony. The limitations of random-based active bat rabies surveillance over passive bat rabies surveillance and its possible application of targeted approaches for future research activities on bat lyssavirus dynamics and maintenance are discussed.

Key words: Bat rabies, epidemiology, lyssavirus, surveillance.

\section{INTRODUCTION}

Rabies is one of the most important zoonotic diseases in the world [1]. The causative agents are

\footnotetext{
* Author for correspondence: Dr C. M. Freuling, Institute of Molecular Biology, Friedrich-Loeffler-Institut, Federal Research Institute for Animal Health, Südufer 10, 17493 Greifswald-Insel Riems, Germany.

(Email: conrad.freuling@fli.bund.de)
}

negative-strand RNA viruses of the genus Lyssavirus of the family Rhabdoviridae [2]. Today, 15 different lyssavirus species are known to occur worldwide and except for Mokola virus (MOKV) and Ikoma virus (IKOV) all of them were found in bats suggesting that species from the order Chiroptera represent the true reservoir hosts for lyssaviruses [3]. While rabies in carnivores has been known for millennia and was the subject of intensive research, our 
understanding of rabies in bats concerning, e.g. epidemiology, prevalence, distribution and pathogenicity is still incomplete [4]. This is due to the fact that in contrast to terrestrial rabies, bat rabies has only been known for the past 100 years [3]. In addition, bat rabies may be driven by different mechanisms [3]. Furthermore, the nocturnal and often cryptic activity of bats limited studies on the occurrence of lyssavirus infections and their dynamics and maintenance in relation to bat biology [5].

So far, five out of the 15 known bat-associated lyssavirus species have been detected in Europe. The great majority of European bat rabies cases detected between 1977 and $2012(n=1033$, Rabies Bulletin Europe, www.who-rabies-bulletin.org) were caused by European bat lyssavirus type 1 (EBLV-1) and type 2 (EBLV-2) (for review see [6]). While EBLV-1 is associated with Eptesicus spp. (Serotine bat - E. serotinus, Isabelline Serotine bat-E. isabellinus), EBLV-2 has been isolated from Myotis spp. (Daubenton's bat $-M$. daubentonii, Pond bat $-M$. dasycneme). Consequently, these bat species were considered as the only reservoir hosts for bat-associated lyssaviruses in Europe. However, recently novel bat lyssaviruses have been detected in other European insectivorous bats. Bokeloh bat lyssavirus (BBLV) was detected in Natterer's bats ( $M$. nattereri) in Germany and France in 2010 and 2012 [7, 8], whereas West Caucasian bat lyssavirus (WCBV) and Lleida bat lyssavirus (LLEBV) were found in Schreiber's bent-winged bats (Miniopterus schreibersii) in the West Caucasian Mountains and on the Iberian Peninsula in 2003 and 2012, respectively $[9,10]$. It is unknown whether the latter two viruses use the same bat species as a reservoir, or whether the geographically distant populations represent two distinct sibling species of Mi. schreibersii.

In contrast to other parts of the world, all 52 European bat species are classified as endangered and, therefore, are protected by the regulations of Council Directive 92/43/EEC of the European Union on the Conservation of Bats in Europe [11] or by national legislation, which limits surveillance efforts. Although guidelines for active and passive bat rabies surveillance have been established by the European research consortium, Med-Vet-Net [12], and were later adopted by EUROBATS [13], the level of bat rabies surveillance in Europe is still very heterogeneous [6]. Passive surveillance as the method of choice focuses on investigations of bats which were found with suspicious clinical symptoms or dead, whereas active surveillance for bat lyssavirus infections consisting of collecting and investigating micro-samples of blood and saliva from free-living indigenous bat populations as pioneered in North America [14-16] is considered valuable for obtaining additional information. As a consequence, active bat rabies surveillance in free-ranging bat populations was implemented in the UK [17, 18], France [19], Spain [20-24], Switzerland [25], Sweden [26], Slovenia [27, 28], Serbia [29] and Belgium [30]. While in some countries results from passive surveillance were confirmed by active surveillance, the detection of virus neutralizing antibodies (VNA) in sera of insectivorous bats indicated the presence of lyssaviruses in countries where bat rabies cases had not yet been reported [6] resulting in speculation as to whether bat lyssavirus infections are in fact more frequent and more widely distributed than assumed from passive surveillance data.

Since Germany is among the countries with the highest frequency of bat rabies cases in Europe ([6], Rabies Bulletin Europe, www.who-rabies-bulletin. org) active bat rabies surveillance was initiated in order to yield additional data on the distribution of bat lyssaviruses known to circulate in German bat populations, e.g. EBLV-1, EBLV-2, and BBLV $[6,7]$. The objective of this study was to test whether active bat rabies surveillance can (i) complement passive surveillance, (ii) assist in generating new data/ information on the occurrence of bat-associated lyssavirus infections in indigenous bat populations, and (iii) become a tool for wider application. Here, we present the data and conclusions from two decades of active bat rabies surveillance in Germany.

\section{MATERIAL AND METHODS}

\section{Sampling}

From 1993 until 2012, free-ranging bats of different species were captured at 42 different locations in seven German federal states, i.e. Bavaria, Berlin, Brandenburg, Hesse, Mecklenburg-Western Pomerania, Saxony and Saxony-Anhalt, during routine mark-capture-recapture studies in close collaboration with local bat biologists (see Supplementary Fig. S1, available online). Capture sites were selected either randomly or based on established long-time monitoring of indigenous bat species. None of the colonies had a history of confirmed bat rabies cases. Bats were caught between May and September, either during night-time using mist nets or harp traps at the 
cave entrances of swarming sites, maternity roosts in buildings and hunting areas over bodies of flowing or standing water, or during routine inspection of artificial maternity roosts in forests. Occasionally, bats were also sampled during hibernacula (October-February).

Species of bats were identified by bat biologists using morphological features [31, 32], and data on sex, age, reproductive status, forearm length, and weight were collected. Prior to release, bats were marked with a uniquely numbered bat ring for individual identification (Fledermausmarkierungszentrale Sächsisches Landesamt für Umwelt und Geologie, Dresden, Germany; Beringungszentrale-Zoologisches Forschungsmuseum Alexander Koenig, Bonn, Germany).

To detect viral RNA or infectious virus in the saliva of bats, oropharyngeal swab samples were taken using dry sterile cotton swabs (Nerbe plus $\mathrm{GmbH}$, Germany). Swabs were placed into $500 \mu 1$ sterile minimum essential medium (MEM-10, with 2\% streptomycin). Blood of sub-adult and adult bats was taken by puncture of the antebrachial vein [33] or the vein in the uropatagium using a 26-gauge needle and collected with a pipette into a $1 \mathrm{ml}$ Eppendorf tube. For animal welfare reasons bleeding of bats was omitted in the case of small bats, poor general health conditions, and signs of weakness or malnutrition. Moreover, if no blood could be obtained after venepuncture further attempts were abandoned. After sampling bats were offered $10 \%$ glucose solution as replenishment. All samples were instantly stored at $\approx 4{ }^{\circ} \mathrm{C}$ in cool boxes in the field and transferred the same day to the laboratory. Here, oropharyngeal swabs were spun down at $1000 \mathrm{rpm}$ to retrieve absorbed medium from the cotton swab. Serum was extracted from blood samples after centrifugation at $1000 \mathrm{rpm}$ for $10 \mathrm{~min}$. Subsequently, oropharyngeal swabs and serum samples were stored at $-80^{\circ} \mathrm{C}$ until testing. Capturing, handling, ringing, and sampling of bats was done under supervision of bat biologists following guidelines approved by the respective competent authorities.

\section{Laboratory tests}

\section{Polymerase chain reaction ( $P C R$ )}

RNA from oropharyngeal swab samples $(250 \mu \mathrm{l})$ was extracted either using commercial guanidinethiocyanate-based RNA extraction followed by column-based purification (RNeasy Mini kit, Qiagen, Germany), TRIzol ${ }^{\circledR}$ (Invitrogen ${ }^{\mathrm{TM}}$ ) or peqGOLD TriFast $^{\mathrm{TM}}$ (Peqlab Biotechnologie GmbH, Germany) according to manufacturers' recommendations. RNA was re-suspended in a volume of $20 \mu$ ldistilled water and stored at $-80^{\circ} \mathrm{C}$ until testing. Amplification and detection of viral RNA was undertaken using two different reverse transcriptase-polymerase chain reaction (RT-PCR) methods specific for EBLV-1 and EBLV-2. While from 1993 to 2004 oropharyngeal swab samples were tested by nested RT-PCR as described previously [34], between 2005 and 2012 samples were investigated by a TaqMan-based quantitative real-time PCR (qRT-PCR) using primers and probes as shown in Supplementary Table S1 (available online). For the duplex EBLV-1 and EBLV-2 assay, a master mix consisting of $3.25 \mu \mathrm{l}$ RNase-free water, $12.5 \mu \mathrm{l} 5 \times$ QuantiTect Virus NR master mix, $0 \cdot 25 \mu \mathrm{l}$ $100 \times$ QuantiTect Virus RT mix (Qiagen, Germany), $2 \cdot 0 \mu \mathrm{l}$ specific primer-probe mix each for EBLV-1 and EBLV-2 (10 pmol EBLV-specific primers +5 pmol EBLV-specific probes) for one reaction was prepared and $5 \mu \mathrm{l}$ RNA template was added. For amplification the following temperature profile was used: $30 \mathrm{~min}$ at $50{ }^{\circ} \mathrm{C}$ (reverse transcription), $15 \mathrm{~min}$ at $95^{\circ} \mathrm{C}$ (inactivation reverse transcriptase/activation Taq polymerase), followed by 42 cycles of $30 \mathrm{~s}$ at $95^{\circ} \mathrm{C}$ (denaturation), $30 \mathrm{~s}$ at $55^{\circ} \mathrm{C}$ (annealing) and $30 \mathrm{~s}$ at $72^{\circ} \mathrm{C}$ (elongation).

Following the discovery of BBLV in 2010 [7], remaining samples of Myotis species (e.g. M. nattereri) were additionally tested using a BBLV-specific qRT-PCR as described previously [35] (Supplementary Table S1). Positive control and negative control (water) samples were analysed in parallel in each PCR run. For the real-time PCRs $C_{t}$ values $>35$ were considered negative. RNA samples from oropharyngeal swabs were pooled $(n=4)$ and, in case of positive results, individual RNA samples were retested separately.

\section{Rabies tissue culture infection test (RTCIT)}

Virus isolation was only performed from RT-PCRpositive oropharyngeal swab samples using RTCIT [36]. Briefly, mouse neuroblastoma cells (MNA 42/ 13; Friedrich-Loeffler-Institut, Germany) were inoculated with $200 \mu \mathrm{l}$ of the remaining oropharyngeal swab medium and incubated for 3 days at $37^{\circ} \mathrm{C}$ and $5 \% \mathrm{CO}_{2}$. A result was confirmed negative after the third consecutive cell passage. 


\section{Sequence analysis}

RTCIT-positive samples were further characterized using sequence analysis of the $\mathrm{N}$-coding region. Sequencing of the nucleoprotein gene was performed with a panel of IRD-800-labelled forward and reverse primers as described previously [37]. Sequence analysis was performed using the Lasergene 6 package (DNAstar Inc., USA).

\section{Rapid fluorescent focus inhibition test (RFFIT)}

Individual serum samples $(25 \mu \mathrm{l})$ were tested for the presence of VNA using a modified RFFIT as described previously [38] with an EBLV-1 isolate as test virus [39]. In general, sera were tested in twofold serial dilutions on mouse neuroblastoma cells (MNA 42/13) with a starting dilution of 1:10. If there was not a sufficient amount of serum available, sera were tested with a starting dilution of 1:20. A heterologous WHO international standard immunoglobulin (second human rabies immunoglobulin preparation, National Institute for Standards and Control, UK) adjusted to $1.5 \mathrm{IU} / \mathrm{ml}$ served as a positive control. The VNA titre was expressed as the reciprocal of the serum dilution showing a 50\% reduction in fluorescent foci of the EBLV-1 test virus in vitro.

\section{RESULTS}

From 1993 until 2012, bats were caught at 42 different locations (Supplementary Fig. S1). In four capture sites in Brandenburg and Saxony-Anhalt sampling was conducted repeatedly (up to six times) on an annual basis, while at the remaining locations capturing was only conducted once. During the study period, a total of 4546 oropharyngeal swabs were taken from 18/24 bat species indigenous to Germany. The majority of samples were collected in Saxony-Anhalt $(n=3118)$ and Brandenburg $(n=530)$. The most frequently sampled species were the noctule bat (Nyctalus noctula) and Natterer's bat ( $M$. nattereri), followed by Daubenton's bat $(M$. daubentonii) and Nathusius' pipistrelle bat (Pipistrellus nathusii) (Table 1). Of the 4546 oropharyngeal swabs taken, $1628(35.8 \%)$ were screened using RT-PCR on the presence of all three European bat lyssaviruses known to exist in Germany (EBLV-1, EBLV-2, BBLV), while $2312(50 \cdot 9 \%)$ samples were tested for viral RNA of both types of EBLVs. A total of 304, 269 and 33 oropharyngeal swabs were investigated exclusively for EBLV-1-, EBLV-2- and BBLV-specific RNA, respectively. This resulted in a total of 4277 , 4209 and 1661 individual EBLV-1-, EBLV-2-, and BBLV-specific RT-PCR tests (Table 1).

EBLV-2- and BBLV-specific RNA was not detected in 4209 and 1661 tested swabs, respectively, whereas by RT-PCR EBLV-1-specific amplicons were obtained in seven of 4277 samples representing five serotine bats (E. serotinus), one Natterer's bat ( $M$. nattereri) and one Western barbastelle bat (Barbastella barbastellus) (Table 1). Of those seven RT-PCR-positive oropharyngeal swab samples tested by RTCIT, viable EBLV-1 was isolated from a single serotine bat that was captured in a maternity roost in the village of Tornow, Brandenburg, in 1993. Sequence analysis of this particular EBLV-1 isolate (laboratory no. 31448, GenBank accession no. KF042302) showed a $99 \cdot 9 \%$ identity in a $1356 \mathrm{bp}$ fragment of the $\mathrm{N}$ gene to another EBLV-1 isolate (laboratory no. 11647, GenBank accession no. KF042303) from a rabid serotine bat obtained during passive bat rabies surveillance in 2005 from the city of Kyritz, Brandenburg, $<10 \mathrm{~km}$ from the first sampling site. Attempts to obtain larger nucleoprotein gene-specific amplicons of the remaining six qRT-PCR-positive samples using conventional RT-PCR for subsequent sequencing failed.

Of the 4546 bats sampled during the study period, a total of $1736(36 \cdot 2 \%)$ blood samples from 13 different bat species were collected with volumes usually ranging from $3 \mu \mathrm{l}$ to $200 \mu \mathrm{l}$, equalling $1 \cdot 5-100 \mu \mathrm{l}$ of serum. From individuals of the larger bat species, e.g. E. serotinus, M. myotis and N. noctula, up to $500 \mu \mathrm{l}$ of blood ( $250 \mu \mathrm{l}$ serum) could be obtained. For $510(29 \cdot 4 \%)$ bats, the amount of serum was insufficient $(25 \mu \mathrm{l})$ to be serologically analysed (Table 1). Of the 1226 sera tested against EBLV-1 using modified RFFIT, $85 \cdot 6 \%$ of samples had VNA titres of $<1: 10$. Virus neutralizing activity against EBLV-1 with titres $\geqslant 1: 10$ was detected in $146(11.9 \%)$ sera from eight bat species, while $31(2 \cdot 5 \%)$ sera from four bat species, e.g. E. serotinus, M. myotis, B. barbastellus, N. noctula, exhibited titres $\geqslant 1: 20$ (Table 1).

Three qRT-PCR-positive oropharyngeal swabs and 18 sera with VNA titres $\geqslant 1: 10$ originated from an E. serotinus maternity colony in the village of Hartmannsdorf, Brandenburg, consisting of about 30-60 individuals (Supplementary Fig. S1, Table 2). Of the 83 bats captured from this colony at six different time points between 2002 and 2007, a total of 27 individuals were recaptured and resampled twice $(n=11)$ or three times $(n=6)$. Overall, 17 individuals 
Table 1. Screening results of 18 indigenous bat species sampled in Germany between 1993 and 2012 for the presence of lyssavirus-specific RNA (oropharyngeal swabs) and VNAs (serum samples) using RT-PCR and modified RFFIT, respectively

\begin{tabular}{|c|c|c|c|c|c|c|c|c|c|c|c|c|}
\hline \multirow[b]{3}{*}{ Bat species } & \multicolumn{4}{|c|}{ Oral swab samples tested for } & \multicolumn{8}{|c|}{ Serum samples tested for EBLV-1 } \\
\hline & \multicolumn{2}{|c|}{ EBLV-1 } & \multirow{2}{*}{$\frac{\text { EBLV-2 }}{N}$} & \multirow{2}{*}{$\frac{\mathrm{BBLV}}{N}$} & \multirow{2}{*}{$\frac{\text { Sampled }}{N}$} & \multirow{2}{*}{$\frac{\text { Tested }}{N}$} & \multicolumn{6}{|c|}{ VNA titre } \\
\hline & $N$ & Pos. & & & & & $<1: 10$ & $<1: 20$ & $\geqslant 1: 10$ & $\geqslant 1: 20$ & $\geqslant 1: 40$ & $\geqslant 1: 80$ \\
\hline B. barbastellus & 105 & 1 & 83 & 3 & 58 & 36 & 10 & 8 & 11 & 7 & & \\
\hline E. nilssonii & 40 & & 40 & 4 & 5 & 0 & & & & & & \\
\hline E. serotinus & 286 & 5 & 259 & 6 & 226 & 174 & 133 & 12 & 18 & 3 & 4 & 4 \\
\hline M. alcathoe & 10 & & 10 & 6 & & & & & & & & \\
\hline M. bechsteinii & 94 & & 92 & 48 & 3 & 1 & 1 & & & & & \\
\hline M. brandtii & 187 & & 186 & 152 & 4 & 0 & & & & & & \\
\hline M. dasycneme & 1 & & 1 & & & & & & & & & \\
\hline M. daubentonii & 387 & & 654 & 74 & 4 & 0 & & & & & & \\
\hline M. myotis & 431 & & 431 & 287 & 336 & 231 & 208 & & 17 & 2 & 3 & 1 \\
\hline M. mystacinus & 43 & & 42 & 35 & & & & & & & & \\
\hline M. nattereri & 717 & 1 & 679 & 696 & 92 & 23 & 15 & & 8 & & & \\
\hline N. leisleri & 256 & & 253 & 3 & 202 & 106 & 99 & & 7 & & & \\
\hline$N$. noctula & 876 & & 691 & 48 & 756 & 627 & 571 & 9 & 40 & 7 & & \\
\hline P. nathusii & 433 & & 395 & 88 & 18 & 5 & 2 & & 3 & & & \\
\hline P. pipistrellus & 54 & & 53 & 15 & & & & & & & & \\
\hline P. pygmaeus & 193 & & 193 & 164 & & & & & & & & \\
\hline Pl. auritus & 161 & & 144 & 30 & 17 & 9 & 8 & 1 & & & & \\
\hline Pl. austriacus & 3 & & 3 & 2 & 15 & 14 & 3 & & 11 & & & \\
\hline Total & 4277 & 7 & 4209 & 1661 & 1736 & 1226 & 1050 & 30 & 115 & 19 & 7 & 5 \\
\hline
\end{tabular}

VNA, Virus neutralization antibody; RT-PCR, reverse transcriptase-polymerase chain reaction; RFFIT, rapid fluorescent focus inhibition test.

were either qRT-PCR positive or had VNA titres $\geqslant 1: 10$. Two qRT-PCR-positive bats (A 42019, A42 $039)$ exhibited VNA titres $<1: 10$ at the time of first sampling, of which one showed a substantial increase in neutralizing activity 1 year later. The serotine bat with ID A 42046 was the only individual that had an initial VNA titre of 1:10 in 2003 which increased fourfold when testing qRT-PCR positive 1 year later. In five individuals a decline in VNA titres was observed over time, whereas in three bats titres increased (Table 2).

\section{DISCUSSION}

Although during the past 35 years $>1000$ bat rabies cases were reported in Europe [6], bat rabies surveillance is still very heterogeneous in terms of existing networks of bat biologists, the number of bat species submitted and individual bats investigated. Some European bat species have never been investigated for rabies, thus their role in the epidemiology of lyssaviruses remains elusive [6]. The discovery of the novel lyssavirus species BBLV and LLEBV in Natterer's bats $(M$. nattereri) and Schreiber's bent-winged bats (Mi. schreibersii), respectively [7, 9], highlights that besides Serotine (E. serotinus, E. isabellinus), Daubenton's ( $M$. daubentonii) and Pond ( $M$. dasycneme) bats, additional bat species could serve as reservoirs for known or still unknown lyssavirus species in Europe. To obtain more information on the occurrence of bat-associated lyssaviruses, active bat rabies surveillance has been initiated in the Americas [14-16]. Following successful detection of rabies virus (RABV)specific antigen and VNAs in free-ranging American bat populations, active bat rabies surveillance was also initiated in Europe to obtain a better picture of the actual incidence and prevalence of bat rabies. The use of molecular techniques, e.g. RT-PCR, for the detection of bat lyssavirus-specific RNA was first applied in Europe [24]. So far, active bat rabies surveillance has been conducted in eight European countries (for review see [6]) including Germany, which implemented active bat rabies surveillance of free-ranging bat colonies in addition to the already 
Table 2. Results of $R T-P C R$ and serological testing of 17 individual serotine bats (E. serotinus) originating from a maternity colony in Hartmannsdorf, Brandenburg, that were either qRT-PCR positive (oropharyngeal swabs) or had VNA titres $\geqslant 1: 10$ by RFFIT when captured or recaptured between 2002 and 2005

\begin{tabular}{|c|c|c|c|c|c|c|c|c|c|c|}
\hline \multirow[b]{2}{*}{ Ring ID } & \multicolumn{2}{|c|}{2002 (July) } & \multicolumn{2}{|c|}{2003 (July) } & \multicolumn{2}{|l|}{2004 (May) } & \multicolumn{2}{|l|}{2004 (July) } & \multicolumn{2}{|l|}{2005 (July) } \\
\hline & RT-PCR & VNA & RT-PCR & VNA & qRT-PCR & VNA & qRT-PCR & VNA & qRT-PCR & VNA \\
\hline A 42010 & - & $\geqslant 1: 80$ & & & & & & & & \\
\hline A 42016 & - & $\geqslant 1: 80$ & & & & $\geqslant 1: 40$ & - & $<1: 10$ & & \\
\hline A 42018 & - & $\geqslant 1: 20$ & & & & & & & & \\
\hline A 42019 & - & $<1: 20$ & & & + & $<1: 10$ & & & - & $<10$ \\
\hline A 42025 & - & $1: 10$ & - & $1: 10$ & & & & & & \\
\hline A 42028 & - & $<1: 20$ & - & $1: 10$ & & & & & & \\
\hline A 42034 & & & - & 1:10 & - & $<1: 10$ & & & & \\
\hline A 42035 & & & - & $<1: 10$ & & & & & - & $\geqslant 1: 40$ \\
\hline A 42038 & & & - & 1:10 & & & - & $<1: 10$ & - & $<1: 10$ \\
\hline A 42039 & & & & & + & $<1: 10$ & & & - & $\geqslant 1: 80$ \\
\hline A 42042 & & & - & $1: 10$ & & & - & $<1: 10$ & - & n.a. \\
\hline A 42044 & & & - & $1: 10$ & & & & & & \\
\hline A 42046 & & & - & $1: 10$ & + & $\geqslant 1: 40$ & & & & \\
\hline A 42047 & & & - & $1: 10$ & & & & & & \\
\hline A 42049 & & & - & $1: 10$ & & & & & & \\
\hline A 42051 & & & - & $1: 10$ & & & & & & \\
\hline A 42052 & & & - & $1: 10$ & & & - & $1: 10$ & - & $<1: 10$ \\
\hline
\end{tabular}

RT-PCR, Reverse transcriptase-polymerase chain reaction; qRT-PCR, quantitative real-time PCR; VNA, virus neutralization antibody; RFFIT, rapid fluorescent focus inhibition test.

established passive bat rabies surveillance system in 1993 [40]. In previous field studies a total of $23 / 52$ known European bat species were tested for lyssavirus infections [6]. During active bat rabies surveillance in Germany, 18/24 indigenous bat species were screened for the presence of EBLV- and BBLV-specific RNA, some of which were underrepresented by both routine and retrospective passive surveillance ([40], J. Schatz et al., unpublished data). While studies in the UK and Spain had mainly focused on reservoir species, i.e. Daubenton's bat (M. daubentonii), and Eptesicus bat species [17, 20], others screened the greater mouse-eared bat (M. myotis) [23], while only in France, Slovenia and in one study from Spain was a similarly high number of different bat species sampled [19, 21, 27]. With more than 4000 oropharyngeal swabs and 1200 blood samples tested by RT-PCR and RFFIT, respectively, this is one of the most extensive active bat rabies surveillance studies ever conducted in Europe.

While in several European studies no EBLVspecific RNA was detected in oropharyngeal swabs [6], we found seven oropharyngeal swab samples positive for EBLV-1 by RT-PCR which is in accordance with previous reports from Spain, UK and Switzerland [13, 20, 24, 25]. The majority of
RT-PCR-positive results were associated with E. serotinus bats, the natural reservoir host of EBLV-1. Of serotine bats, $1.7 \%$ of all oropharyngeal swabs tested positive for viral RNA. Similarly, during a 5-year study in Spain, $2 \cdot 8 \%$ of oropharyngeal swab samples from E. isabellinus yielded viral RNA [20]. At the serotine bat colony level (Hartmannsdorf, Brandenburg) the percentage of qRT-PCR-positive swabs reached $4 \cdot 7 \%$. In contrast, up to $21 \%$ RT-PCR-positive results were reported from a Spanish serotine bat colony targeted after the detection of a bat rabies case during passive surveillance [24]. In our study, repeated detection of viral RNA in saliva swabs from recaptured serotine bats (E. serotinus) was not observed. In Spain, an initially EBLV-1-positive E. isabellinus was negative on recapturing [20], thus supporting observations in experimentally infected serotine bats [41, 42]. However, unlike in other studies, not only serotine bats but also a single Natterer's bat and Western barbastelle bats tested positive for EBLV-1-specific RNA. Interestingly, EBLV-1 RT-PCR-positive brain tissue was reported from a Natterer's bat from Spain [21]. However, repeated detection of the novel BBLV in Natterer's bats in Germany and France [7, 8, 35] suggests that this species may act as reservoir host for this proposed new lyssavirus species and, thus, EBLV-1 
infections in this bat species may represent spillover events. In Germany, E. serotinus, M. nattereri and B. barbastellus hibernate together, e.g. in basement vaults or different types of caves [31]. Additionally, during passive surveillance, EBLV-1 spillover infections were also detected in other bat species in Germany which co-hibernate with E. serotinus [40]. Generally, albeit already a high specificity of the RT-PCR is given through hybridization probes, in the future sequencing of RT-PCR amplicons can confirm the presence of known lyssaviruses or may detect yet unknown variants or novel lyssaviruses.

The isolation of viable bat lyssaviruses during active surveillance appears to be difficult. During capture studies in Spain and Finland, for example, EBLVs were isolated from brain tissue of individual bats that had shown clinical signs suggestive of rabies before dying during handling [24, 43]. Markedly, in our study infectious virus could be isolated from a single RT-PCR-positive serotine bat. Despite the detection of EBLV-specific RNA in very few oropharyngeal swab samples as reported previously [6], to our knowledge, this is the only EBLV-1 isolation from an oropharyngeal swab of a bat obtained during active surveillance in Europe. Interestingly, the bat did not show any clinical symptoms suggestive of rabies at the time of sampling.

The low number of RT-PCR-positive animals corroborates results obtained in experimental studies of EBLV infection in European bats (E. serotinus, M. daubentonii) and in North American big brown bats (E. fuscus), in which virus shedding was rarely detected [41, 42, 44]. However, active bat rabies surveillance studies are considerably biased by random sampling, and samples do not necessarily represent the entire populations of each indigenous bat species. Other reasons for the low number of RT-PCRpositive animals could be the unknown infectious status of the targeted bat colonies, and the fact that not all animals of a colony can be captured at the same time point. Furthermore, bat lyssaviruses are causative agents considered to cause non-persistent infections [4, 5]. While RT-PCR assays generally have a high analytical sensitivity, a dilution effect of viral RNA in the oropharyngeal swab sample and possible degradation of RNA cannot be completely excluded. Although the latter could have been avoided using RNA stabilizing chemicals, virus isolation attempts would then be impossible. Another limitation is the diversity of lyssaviruses circulating in European bats. At the time this study was initiated, only EBLV-1 was known to be present in Germany. In the meantime EBLV-2 and BBLV were detected by passive surveillance $[7,45]$. While the presence of EBLV-2 was also analysed using a combined EBLV-1/ -2 assay, only the more recently collected samples from this study were screened for BBLV-specific RNA. However, this study could not provide further evidence for the presence of BBLV infections in indigenous bats (Table 1). Broader reacting pan-lyssavirus PCR assays with high sensitivity as described, e.g. by Hayman et al. [46], should improve the detection of lyssavirus species during active surveillance.

In our study, 1226 blood samples from 10/18 bat species sampled during the observation period were investigated for the presence of EBLV-specific VNA. In other European efforts, a total of 22 bat species were the subject of serosurveillance with the number of bat species and bats tested in individual studies ranging from 1-14 and 24-976, respectively (Table 3). Similar to previous studies from other European countries, neutralizing activity suggesting the presence of EBLV-specific VNAs was found in eight bat species with a percentage of positives ranging from $7 \cdot 5 \%[95 \%$ confidence interval (CI) $5 \cdot 7-9 \cdot 8]$ to $78 \cdot 6 \%(95 \% \mathrm{CI}$ $52 \cdot 4-92 \cdot 4$ ) at a cut-off of $\geqslant 1: 10$ (Table 1 ). When the higher cut-off was chosen VNAs were only found in four or two bat species, respectively (Table 1). Highest VNA titres against EBLV-1, however, were found in its known reservoir host, i.e. E. serotinus (Table 1). Interestingly, $28 \cdot 6 \%(95 \%$ CI $18 \cdot 4-41 \cdot 5)$ of 56 tested individuals from a single maternity colony in the municipality of Hartmannsdorf, a roosting site with several EBLV-1-positive RNA in oral swabs, had VNA titres of $\geqslant 1: 10$, resulting in a seroprevalence of $0-61 \cdot 1 \%(95 \%$ CI $38 \cdot 6-79 \cdot 7)$ per year. Our data indicate the presence of a significant dynamic in virus transmission and serological response with increasing and decreasing VNA titres in bats in this particular colony (Table 2). This supports findings from serological studies on Lagos bat virus which postulated that acute transmission of bat lyssaviruses in adapted bat hosts occurs at a far higher rate than the occurrence of disease [47]. Our results further support previous experimental studies that although lyssaviruses have the ability to cause clinical disease in bats, they may not necessarily be fatal [41, 42, 44]. Unlike in other mammalian species where antibodies against RABV are only detectable in the final stage of infection, shortly before the animal's death, we were able to observe seroconversion in repeatedly captured bats (Table 2). 
Table 3. Comparison of previous serosurveillance studies conducted in Europe with the study from Germany using different modified versions of virus neutralization assays (modified after Schatz et al. [6])

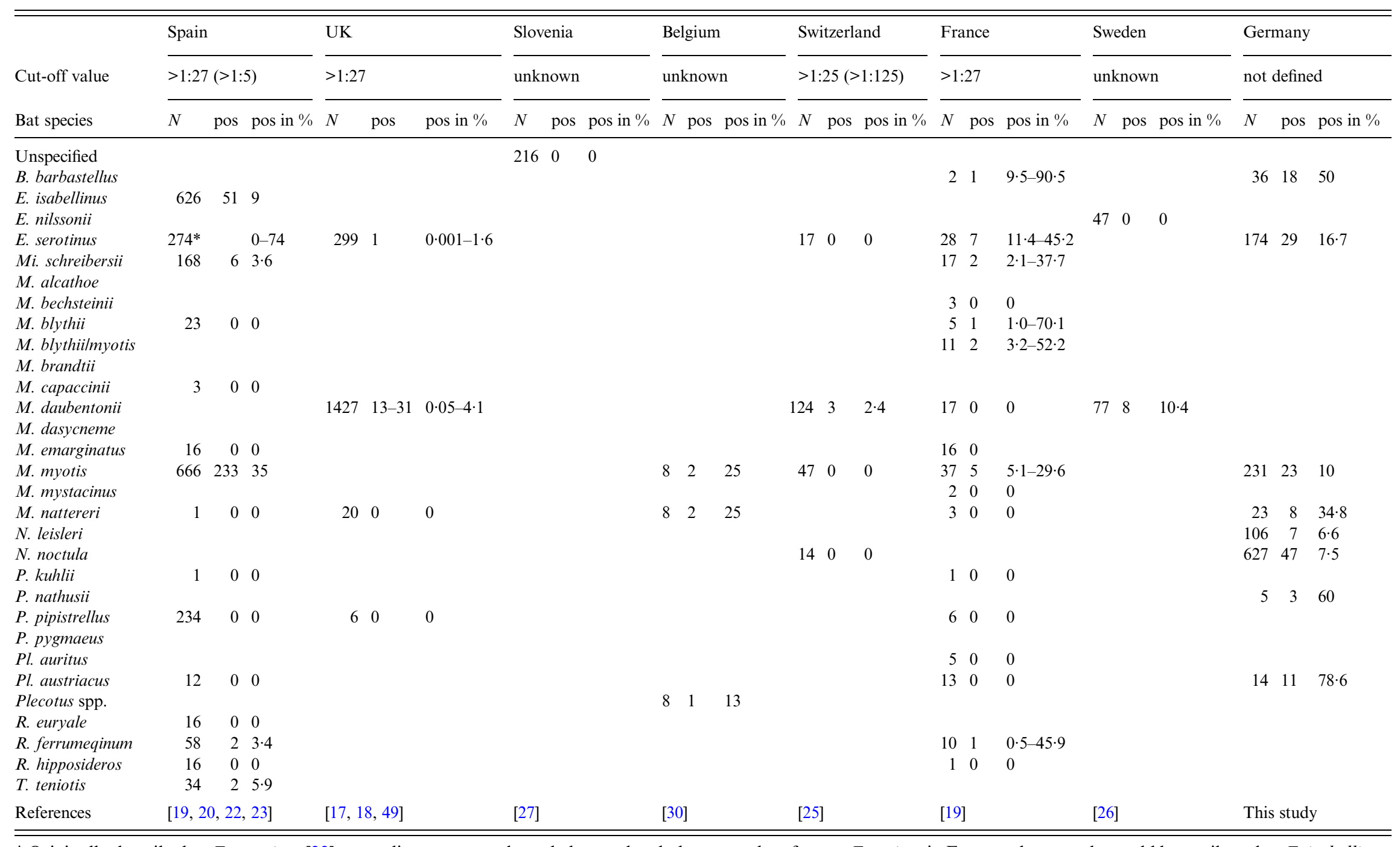

* Originally described as E. serotinus [22], according to present knowledge on the phylogeography of genus Eptesicus in Europe, these results could be attributed to E. isabellinus. 
Interestingly, evidence for the presence of VNAs against EBLV-1 was also found in M. myotis. At a cut-off of $\geqslant 1: 10,9 \cdot 9 \%(95 \%$ CI $6 \cdot 7-14 \cdot 5)$ of the sera are considered positive, while with a cut-off of $>1: 20$, comparable to previous studies, the percentage declines to $2 \cdot 6 \%(95 \%$ CI $1 \cdot 2-5 \cdot 5)$. This is in contrast to reports in which seroprevalences between $22.3 \%$ and $45.0 \%$ were found in Spanish M. myotis colonies [21, 23]. EBLV-1 neutralizing activity was also detected in a single M. myotis from both France and Belgium [19, 30]. In total, $1 \cdot 1 \%(95 \%$ CI $0 \cdot 5-2 \cdot 3)$ of $N$. noctula had VNA titres $\geqslant 1: 20$, whereas a surprising $19 \cdot 4 \%(95 \%$ CI $9 \cdot 8-35 \cdot 0)$ of B. barbastellus sera exhibited high VNA activity. If a cut-off of $\geqslant 1: 10$ was chosen, the seroprevalence even increased to $50 \%(95 \%$ CI $34 \cdot 5-65 \cdot 5)$ (Table 1). Besides our study in which an oropharyngeal swab sample of a Western barbastelle bat (B. barbastellus) also tested EBLV-1 positive, in France a single bat from this species was found to have VNAs [19]. With the detection of EBLV-1 RNA in oropharyngeal swabs, the observed seroconversion probably resulted from spillover infections from the EBLV-1 reservoir host E. serotinus. Another possibility is the circulation of as yet unknown EBLV-1-like virus(es). However, since both in active and passive surveillance the number of investigated individuals of $B$. barbastellus is low [6], this species needs to be further investigated. Sporadic rabies cases in $N$. noctula were reported in Europe, although further information on the virus species is not available [6].

For various reasons it is rather difficult to compare results of serological testing between published studies. Unfortunately, serological testing offers limited insights into past exposures of clinically unsuspicious bats as it is critically dependent on the cut-off values used for the test [48]. Moreover, the serum dilution is a critical point for VNA titre determination. In previous surveys, either three- or fivefold serial dilutions were applied while we used a twofold dilution series. Furthermore, the cut-off values often resembled the starting dilution of sera to be tested; in general a cut-off value of $\geqslant 1: 27$ was considered the threshold of positivity (Table 3 ). In our study we did not define a cut-off value, but instead established the VNA titres as the reciprocal of the serum dilution showing a $50 \%$ reduction of the test virus, as done in a study in Spain [22]. Although in general high VNA titres tend to be specific more often, low titres rather tend to be unspecific by resembling non-humoral neutralization or virucidal effects. Even if high VNA titres were specific, the extraordinary high seroprevalences found in Spanish M. myotis colonies [21, 23], compared to ours, raise further questions. This could be a true representation of regional differences in virus circulation and dynamics; however, an influence of the various neutralization test methods applied cannot be excluded. Further, evidence of natural infections in this particular species in Spain is still lacking [6].

Another critical point is the test virus. As in a previous study [19] we used a modified RFFIT with EBLV-1 as test virus. While this seems appropriate for the established reservoir species $E$. serotinus and $E$. isabellinus, it is much more difficult to decide which test virus to use in non-reservoir species. Generally, seropositivity cannot be attributed to a specific lyssavirus, because of cross-reactivity of antibodies to lyssaviruses within phylogroups [49]. In particular, the recent detection of BBLV in $M$. nattereri exemplifies that the presence of as yet unknown lyssavirus species could account for the observed neutralizing activity in certain bat species other than the assumed reservoir species (Tables 1 and 3). In fact, different test viruses were used in other serosurveillance studies. In UK, serum samples of serotine bats and Daubenton's bats were separately tested against EBLV-1 (E. serotinus) and EBLV-2 (Myotis spp.) [17]. VNAs in two Natterer's bats using EBLV-1 as test virus [50] may indicate the presence of EBLV-1, BBLV or an as yet undiscovered virus from phylogroup I. In Spain, sera of different bat species were investigated using EBLV-1 as test virus. Selected positive sera were additionally tested against challenge virus standard (CVS) and EBLV-2 and because of no neutralization regarded as specific [21]. In Switzerland, serum samples of bats were tested with CVS and/or EBLV-2 as challenge virus. Of three positive Daubenton's bats (reciprocal titre of $\geqslant 27$ ) only one serum tested positive using CVS, while the other two seropositive samples (EBLV-2) tested negative against CVS [25]. The latter results exemplify that even though crossreactivity is assumed for members of one phylogroup, different neutralization activities may be observed, depending on the specificity of VNAs, the titre, and the test viruses used. For instance, when sera of EBLV-1 and EBLV-2 experimentally infected ferrets [34] were serologically investigated against EBLV-1 and EBLV-2, sera had lower neutralizing activity using the heterologous test virus (T. Müller et al., unpublished data). Similar reactions were reported from serological tests with Aravan virus (ARAV) 
and Khujand virus (KHUV) [51]. In this respect we are aware of the fact that by using one test virus only we may have missed seroconversion in other bat species, in particular in Myotis species.

Another problem associated with serological testing of bats is often small volume of serum obtained during bleeding. Even from bigger species, e.g. noctule bats ( $N$. noctula), it was often a challenge to obtain sufficient blood for testing. In our opinion, from an animal welfare point of view, serological investigations of small bat species, e.g. Pipistrellus spp., should be reconsidered. Micro-neutralization assays have been used in several studies to cope with limited serum volume [41, 52]; however, unless those tests have been thoroughly validated for routine use, which is often not the case, multiple testing of samples with different challenge viruses is not practicable. Furthermore, the lack of standardized protocols including arbitrary cut-off values, issues of pooling samples, comparability of serological results, and crossneutralization represent a continuing dilemma for lyssavirus serosurveillance in European bats [6, 53, 54].

\section{CONCLUSIONS}

Although active bat rabies surveillance has been recommended for years [12, 13], based on our experience it provides only limited information. Given their aetiopathology the detection of bat lyssaviruses based on RT-PCR and virus isolation using an active random search in free-living populations of bats is coincidental. Except for the serological results the low number of RT-PCR-positive animals including the EBLV-1 isolate obtained in our study originated from regions in Germany where the presence of bat rabies had already been identified through wellestablished passive surveillance. Serological data with all its current uncertainties, however, may assist in generating new data/information on the occurrence of bat-associated lyssavirus infections in indigenous bat populations and hence, could be useful in complementing passive surveillance. However, the detection of cross-neutralization in bat species other than the known reservoir species raises numerous questions that need urgent clarification; among which is specificity against different known bat lyssaviruses. Based on its current limitations and significant logistic efforts required, random-based active bat rabies surveillance is not likely to become a tool to be applied more widely and therefore cannot replace missing passive bat rabies surveillance. Instead targeted sampling of bat colonies with confirmed bat rabies through passive surveillance may assist in gaining deeper insights into bat lyssavirus dynamic and maintenance.

\section{SUPPLEMENTARY MATERIAL}

For supplementary material accompanying this paper visit http://dx.doi.org/10.1017/S0950268813002185.

\section{ACKNOWLEDGEMENTS}

This study would not have been possible without the continued support and assistance of numerous bat biologists and bat conservationists. We would like to especially thank the members of the Arbeitskreis Fledermäuse Sachsen-Anhalt e.V. for including us in their group and sharing their enthusiasm with us about these fantastic animals. Special thanks go to Jeannette Kliemt, Doris Junghans and Martina Steffen for their skilful technical assistance during all these years. This study was undertaken within the frame of a lyssavirus research network financially supported by the German Ministry for Education and Research (BMBF, grant no. 01KI1016A). The authors are also grateful to the Adolf and Hildegard Isler- Stiftung, Germany, and the Research and Policy for Infectious Disease Dynamics (RAPIDD) programme of the Science and Technology Directorate, US Department of Homeland Security, at the Fogarty International Center, National Institutes of Health.

\section{DECLARATION OF INTEREST}

None.

\section{REFERENCES}

1. World Health Organisation. Expert consultation on rabies, first report. World Health Organisation: Technical Report Series 2005; 931, 1-88.

2. Dietzgen RG, et al. Family Rhabdoviridae. In: King AMQ, Adams MJ, Carstens EB, Lefkowitz EJ, eds. Virus Taxonomy: Classification and Nomenclature of Viruses-Ninth Report of the International Committee on Taxonomy of Viruses. San Diego: Elsevier, 2012, pp. 686-713.

3. Banyard AC, et al. Bat rabies. In: Jackson AC, Wunner W, eds. Rabies. New York: Academic Press, 2013.

4. Vos A, et al. European bat lyssaviruses - an ecological enigma. Acta Chiropterologica 2007; 9: 283-296. 
5. Banyard AC, et al. Bats and lyssaviruses. In: Jackson AC, ed. Advance in Virus Research. Amsterdam: Elsevier, 2011, pp. 239-289.

6. Schatz J, et al. Bat rabies surveillance in Europe. Zoonoses and Public Health 2013; 60: 22-34.

7. Freuling CM, et al. Novel lyssavirus in Natterer's bat, Germany. Emerging Infectious Diseases 2011; 17: 15191522.

8. Picard-Meyer E, et al. Isolation of the novel BBLV lyssavirus in Natterer's bat in France. Bulletin epidemiologique 2012; 55: 25.

9. Ceballos NA, et al. Novel lyssavirus in bat, Spain. Emerging Infectious Diseases 2013; 19: 793-795.

10. Botvinkin AD, et al. Novel lyssaviruses isolated from bats in Russia. Emerging Infectious Diseases 2003; 9: 1623-1625.

11. Anon. Agreement on the conservation of populations of European bats (EUROBATS), 1991.

12. Med Vet Net Working Group. Passive and active surveillance of bat lyssavirus infections. Rabies Bulletin Europe 2005; 29: 5.

13. Anon. Agreement on the conservation of populations of bats in Europe (EUROBATS), Annex 5: bat rabies, 2006.

14. Trimarchi CV, Debbie JG. Naturally occurring rabies virus and neutralizing antibody in two species of insectivorous bats of New York State. Journal of Wildlife Diseases 1977; 13: 366-369.

15. Constantine DG, et al. Rabies in New Mexico cavern bats. Public Health Reports 1968; 83: 303-316.

16. Steece R, Altenbach JS. Prevalence of rabies specific antibodies in the Mexican free-tailed bat (Tadarida brasiliensis mexicana) at Lava cave, New Mexico. Journal of Wildlife Diseases 1989; 25: 490-496.

17. Harris SL, et al. Targeted surveillance for European bat lyssaviruses in English bats (2003-06). Journal of Wildlife Diseases 2009; 45: 1030-41.

18. Brookes SM, et al. European bat lyssavirus in Scottish bats. Emerging Infectious Diseases 2005; 11: 572-578.

19. Picard-Meyer E, et al. Active surveillance of bat rabies in France: a 5-year study (2004-2009). Veterinary Microbiology 2011; 151: 390-395.

20. Vazquez-Moron S, et al. Endemic circulation of European bat lyssavirus type 1 in serotine bats, Spain. Emerging Infectious Diseases 2008; 14: 1263-1266.

21. Serra-Cobo J, et al. European bat lyssavirus infection in Spanish bat populations. Emerging Infectious Diseases 2002; 8: 413-420.

22. Perez-Jorda JL, et al. Lyssavirus in Eptesicus-Serotinus (Chiroptera, Vespertilionidae). Journal of Wildlife Diseases 1995; 31: 372-377.

23. Amengual B, et al. Temporal dynamics of European bat lyssavirus type 1 and survival of Myotis myotis bats in natural colonies. PLoS ONE 2007; 2: 1-7.

24. Echevarria JE, et al. Screening of active lyssavirus infection in wild bat populations by viral RNA detection on oropharyngeal swabs. Journal of Clinical Microbiology 2001; 39: 3678-3683.

25. Megali A, et al. Surveillance for European bat lyssavirus in Swiss bats. Archives in Virology 2010; 155: 1655-62.
26. National Veterinary Institute. Surveillance and control programmes: domestic and wild animals in Sweden 2008, 2009.

27. Hostnik P, et al. Determination of bat lyssavirus in Slovenia. Zdravniski Vestnik 2010; 79: 265-271.

28. Presetnik P, et al.. Sunny new from the sunny side of the Alps-active surveillance for lyssaviruses in bats did not reveal the presence of EBLV in Slovenia. In: 2nd International Berlin Bat Meeting: Bat Biology and Infectious Diseases. Berlin, 19-21 February 2010: Leibniz Institute for Zoo and Wildlife Research, 2010.

29. Vranies N, et al. Passive und active surveillance of lyssavirus in bats in Serbia. In: 2nd International Berlin Bat Meeting: Bat Biology and Infectious Diseases. Berlin, 19-21 February 2010: Leibniz Institute for Zoo and Wildlife Research, 2010, p. 94.

30. Klein F, et al. First clue of circulation of lyssaviruses in bat populations. In: 2nd Symposium: 'Wildlife Diseases Environment and Man'. Belgian Wildlife Disease Society, Brussels, 13 October 2007, p. 45.

31. Dietz C, Helversen O. von, Nill D. Handbuch der Fledermäuse Europas und Nordwestafrikas. Biologie, Kennzeichen, Gefährdung. Stuttgart: Kosmos, 2007.

32. Schober W, Grimmberger E. Die Fledermäuse EuropasKennen, bestimmen, schützen, 2nd edn. Stuttgart Franckh-Kosmos Verlags-GmbH, 1998.

33. Kunz TH, Nagy KA. Methods of energy budget analysis. In: Ecological and Behavioral Methods for the Study of Bats. Washington, DC: Smithsonian Institution Press, 1988, pp. 277-302.

34. Vos A, et al. Susceptibility of ferrets (Mustela putorius furo) to experimentally induced rabies with European bat lyssaviruses (EBLV). Journal of Veterinary Medicine. B, Infectious Diseases and Veterinary Public Health 2004; 51: 55-60.

35. Freuling CM, et al. Molecular diagnostics for the detection of Bokeloh bat lyssavirus in a bat from Bavaria, Germany. Virus Research. Published online: 7 August 2013. doi:10.1016/j.virusres.2013.07.021.

36 Webster WA, Casey GA. Virus isolation in neuroblastoma cell culture. In: Meslin FX, Kaplan MM, Koprowski H, eds. Laboratory Techniques in Rabies, 4th edn. Geneva: World Health Organization, 1996, pp. 93-104.

37. Freuling $\mathbf{C}$, et $\boldsymbol{a l}$. A random grid based molecular epidemiological study on EBLV isolates from Germany. Developments in Biologicals (Basel) 2008; 131: 301309.

38. Smith JS, Yager PA, Baer GM. A rapid reproducible test for determining rabies neutralizing antibody. Bulletin of the World Health Organization 1973; 48: 535-541.

39. Cox JH, Schneider LG. Prophylactic immunization of humans against rabies by intradermal inoculation of human diploid cell culture vaccine. Journal of Clinical Microbiology 1976; 3: 96-101.

40. Müller T, et al. Epidemiology of bat rabies in Germany. Archives of Virology 2007; 152: 273-288.

41. Franka R, et al. Susceptibility of North American big brown bats (Eptesicus fuscus) to infection with 
European bat lyssavirus type 1. Journal of General Virology 2008; 89: 1998-2010.

42. Freuling C, et al. Experimental infection of serotine bats (Eptesicus serotinus) with European bat lyssavirus type 1a. Journal of Genral Virology 2009; 90: 2493-2502.

43. Jakava-Viljanen M, et al. First encounter of European bat lyssavirus type 2 (EBLV-2) in a bat in Finland. Epidemiology and Infection 2010; 138: 1581-1585.

44. Johnson N, et al. Experimental study of European bat lyssavirus type-2 infection in Daubenton's bats (Myotis daubentonii). Journal of General Virology 2008; 89: 2662-2672.

45. Freuling CM, et al. First isolation of EBLV-2 in Germany. Veterinary Microbiology 2008; 131: 26-34.

46. Hayman DTS, et al. A universal real-time assay for the detection of lyssaviruses. Journal of Virological Methods 2011; 177: 87-93.

47. Hayman DTS, et al. Endemic Lagos bat virus infection in Eidolon helvum. Epidemiology and Infection 2012; 140: 2163-2171.

48. Bowen RA, et al. Prevalence of neutralizing antibodies to rabies virus in serum of seven species of insectivorous bats from colorado and new Mexico, United States. Journal of Wildlife Diseases 2013; 49: 367-374.

49. Horton DL, et al. Quantifying antigenic relationships among the lyssaviruses. Journal of Virology 2010; 84: 11841-11848.

50. SNH. Scottish Natural Heritage releases latest bat lyssavirus monitoring results. (http://wwwsnhorguk/press/ detailasp?id=2104 2009).

51. Hanlon CA, et al. Efficacy of rabies biologics against new lyssaviruses from Eurasia. Virus Research 2005; 111: 44-54.

52. Andrulonis JA, et al. A micromethod for measuring rabies-neutralizing antibody. Journal of Wildlife Diseases 1976; 12: 552-554.

53. Freuling C, et al. Bat rabies - a Gordian knot? Berliner und Münchener Tierärztliche Wochenschrift 2009; 122: 425-433.

54. Gilbert AT, et al. Deciphering serology to understand the ecology of infectious diseases in wildlife. Ecohealth. Published online: 6 August 2013. doi:10.1007/s10393013-0856-0. 